\title{
Gauge-Higgs Unification: Stable Higgs Bosons as Cold Dark Matter ${ }^{1}$
}

\author{
Yutaka Hosotani \\ Department of Physics, Osaka University \\ Toyonaka, Osaka 560-0043, Japan
}

\begin{abstract}
In the gauge-Higgs unification the 4D Higgs field becomes a part of the extra-dimensional component of the gauge potentials. In the $S O(5) \times U(1)$ gauge-Higgs unification in the Randall-Sundrum warped spacetime the electroweak symmetry is dynamically broken through the Hosotani mechanism. The Higgs bosons become absolutely stable, and become the dark matter of the universe. The mass of the Higgs boson is determined from the WMAP data to be about $70 \mathrm{GeV}$.
\end{abstract}

Keywords: Higgs boson, dark matter, gauge-Higgs unification, Hosotani mechanism.

\section{Introduction}

Does the Higgs boson exist? What is it really like? What constitutes the dark matter in the universe? These are two of the most important problems in current physics. We would like to point out that these two mystery particles are really the same. In the gauge-Higgs unification scenario Higgs bosons become absolutely stable, and become the dark matter of the universe.

In the gauge-Higgs unification the 4D Higgs boson is identified with a part of the extra-dimensional component of the gauge potentials. Its couplings with others particles are controlled by the gauge principle. The $4 \mathrm{D}$ Higgs field corresponds to $4 \mathrm{D}$ fluctuations of an $\mathrm{AB}$ phase in the extra dimensions. [1, 2, 3] In the $S O(5) \times$ $U(1)$ gauge-Higgs unification model in the Randall-Sundrum warped space the $\mathrm{AB}$ phase $\theta_{H}$ takes exactly the value $\frac{1}{2} \pi$ in the vacuum as a consequence of quantum dynamics. At this particular value of $\theta_{H}$ the $4 \mathrm{D}$ Higgs boson becomes absolutely stable.

The relic abundance of the Higgs bosons in the present universe is evaluated definitively with the mass of the Higgs boson as the only relevant variable parameter. Astonishingly the average mass density of the dark matter determined from the WMAP data is obtained with the Higgs mass around $70 \mathrm{GeV}$. It does not contradict with the LEP2 bound, because the $Z Z H$ coupling vanishes at $\theta_{H}=\frac{1}{2} \pi$. The gauge-Higgs unification scenario gives a completely new viewpoint for the Higgs boson. 4] Further it gives definitive predictions for electroweak gauge couplings of quarks and leptons, which can be tested experimentally. [5]

\footnotetext{
${ }^{1}$ To appear in the Proceedings of "SCGT09: Strong Coupling Gauge Theories in LHC Era", Nagoya, Japan, 8-11 December, 2009.
} 


\section{2. $S O(5) \times U(1)$ gauge-Higgs unification in RS}

We consider an $S O(5) \times U(1)$ gauge theory in the five-dimensional RandallSundrum (RS) warped spacetime. [4-14] Its metric is given by

$$
d s^{2}=e^{-2 \sigma(y)} \eta_{\mu \nu} d x^{\mu} d x^{\nu}+d y^{2},
$$

where $\eta_{\mu \nu}=\operatorname{diag}(-1,1,1,1), \sigma(y)=\sigma(y+2 L)$, and $\sigma(y)=k|y|$ for $|y| \leq L$. The fundamental region in the fifth dimension is given by $0 \leq y \leq L$. The Planck brane and the $\mathrm{TeV}$ brane are located at $y=0$ and $y=L$, respectively. The bulk region $0<y<L$ is an anti-de Sitter spacetime with a cosmological constant $\Lambda=-6 k^{2}$.

The RS spacetime has the same topology as the orbifold $M^{4} \times\left(S^{1} / Z_{2}\right)$. Vector potentials $A_{M}(x, y)$ of the gauge group $S O(5)$ and $B_{M}(x, y)$ of $U(1)$ satisfy the orbifold boundary conditions

$$
\begin{gathered}
\left(\begin{array}{c}
A_{\mu} \\
A_{y}
\end{array}\right)\left(x, y_{j}-y\right)=P_{j}\left(\begin{array}{c}
A_{\mu} \\
-A_{y}
\end{array}\right)\left(x, y_{j}+y\right) P_{j}^{-1}, \\
\left(\begin{array}{c}
B_{\mu} \\
B_{y}
\end{array}\right)\left(x, y_{j}-y\right)=\left(\begin{array}{c}
B_{\mu} \\
-B_{y}
\end{array}\right)\left(x, y_{j}+y\right) \\
P_{j}=\operatorname{diag}(-1,-1,-1,-1,+1), \quad(j=0,1),
\end{gathered}
$$

where $y_{0}=0$ and $y_{1}=L$. By the boundary conditions the $S O(5) \times U(1)$ symmetry is reduced to $S O(4) \times U(1) \simeq S U(2)_{L} \times S U(2)_{R} \times U(1)$. The symmetry $S U(2)_{R} \times$ $U(1)$ is further spontaneously broken by a scalar field $\Phi(x)$ on the Planck brane to $U(1)_{Y} . \Phi(x)$ belongs to $\left(0, \frac{1}{2}\right)$ representation of $S U(2)_{L} \times S U(2)_{R}$. The pattern of the symmetry reduction[11, 13] is depicted in fig. 1.

With the above boundary conditions the zero modes of $4 \mathrm{D}$ gauge fields reside in the 4-by-4 matrix part of $A_{\mu}$, whereas the zero modes of $A_{y}$ in the off-diagonal part of $A_{y}$. The latter is an $S O(4)$ vector, or an $S U(2)_{L}$ doublet, corresponding to the Higgs doublet in the standard model. See fig. 2

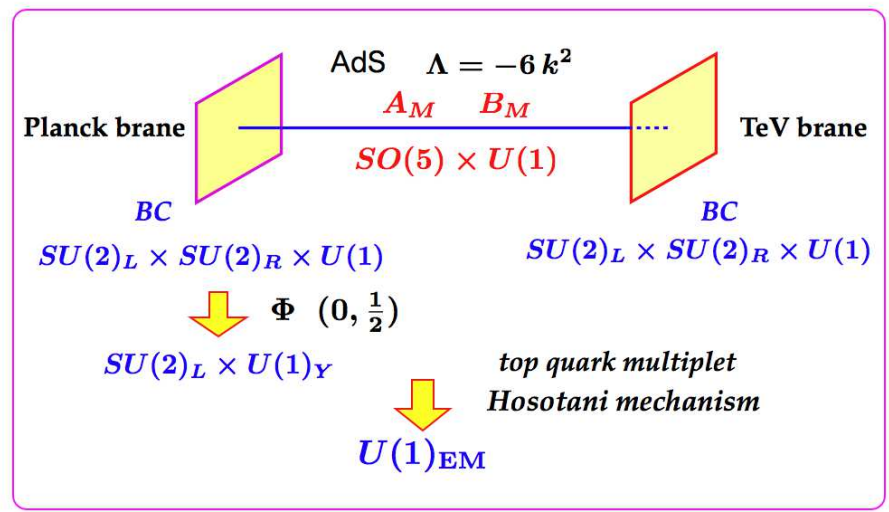

Figure 1: The symmetry reduction in the $S O(5) \times U(1)$ gauge-Higgs unification in RS. 


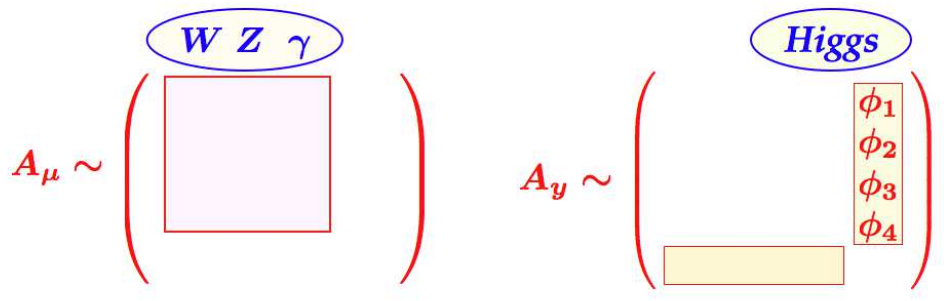

Figure 2: $W, Z, \gamma$ appear as zero modes of $A_{\mu}$ whereas the 4D Higgs field appears as a zero mode of $A_{y}$.

Bulk fermions for quarks and leptons are introduced as multiplets in the vectorial representation of $S O(5)$. [5, 14 In the quark sector two vector multiplets are introduced for each generation. In the lepton sector it suffices to introduce one multiplet for each generation to describe massless neutrinos, whereas it is necessary to have two multiplets to describe massive neutrinos. Each vector multiplet $\Psi_{a}$ is decomposed to $\left(\frac{1}{2}, \frac{1}{2}\right) \oplus(0,0)$ in the $S U(2)_{L} \times S U(2)_{R}$. They satisfy the orbifold boundary condition

$$
\Psi_{a}\left(x, y_{j}-y\right)=P_{j} \Gamma^{5} \Psi_{a}\left(x, y_{j}+y\right),
$$

which gives rise to chiral fermions as zero modes. The left-handed components of $\left(\frac{1}{2}, \frac{1}{2}\right)$ and the right-handed components of $(0,0)$ have zero modes.

In addition to the bulk fermions, right-handed brane fermions $\hat{\chi}_{\alpha}$ are introduced on the Planck brane. The brane fermions $\hat{\chi}_{\alpha}$ belong to the $\left(\frac{1}{2}, 0\right)$ representation of $S U(2)_{L} \times S U(2)_{R}$. These brane fermions are necessary both to have the realistic quark-lepton spectrum at low energies and to have the cancellation of $4 \mathrm{D}$ chiral anomalies associated to the $S O(4) \times U(1)$ gauge fields. The matter content is summarized in fig. 3 The $S O(4) \times U(1)$ gauge invariance is maintained on the Planck brane as well. The bulk fermions $\Psi_{a}$, the brane fermions $\hat{\chi}_{\alpha}$, and the brane scalar $\Phi$ form $S O(4) \times U(1)$ invariant interactions. When $\Phi$ develops a non-vanishing expectation value to spontaneously break $S U(2)_{R} \times U(1)$ to $U(1)_{Y}$, it simultaneously gives mass couplings between $\Psi_{a}$ and $\hat{\chi}_{\alpha}$ which, in turn, make all exotic fermions heavy.

\section{Higgs boson as an AB phase}

As shown in fig. 2, the 4D Higgs field appears as a zero mode of $A_{y}$. Without loss of generality one can suppose that the $A_{y}^{45}$ component develops a nonvanishing expectation value. We write

$$
A_{y}(x, y)=\hat{\theta}_{H}(x) \cdot \sqrt{\frac{4 k}{z_{L}^{2}-1}} h_{0}(y) \cdot T^{\hat{4}}+\cdots, \quad z_{L}=e^{k L}
$$




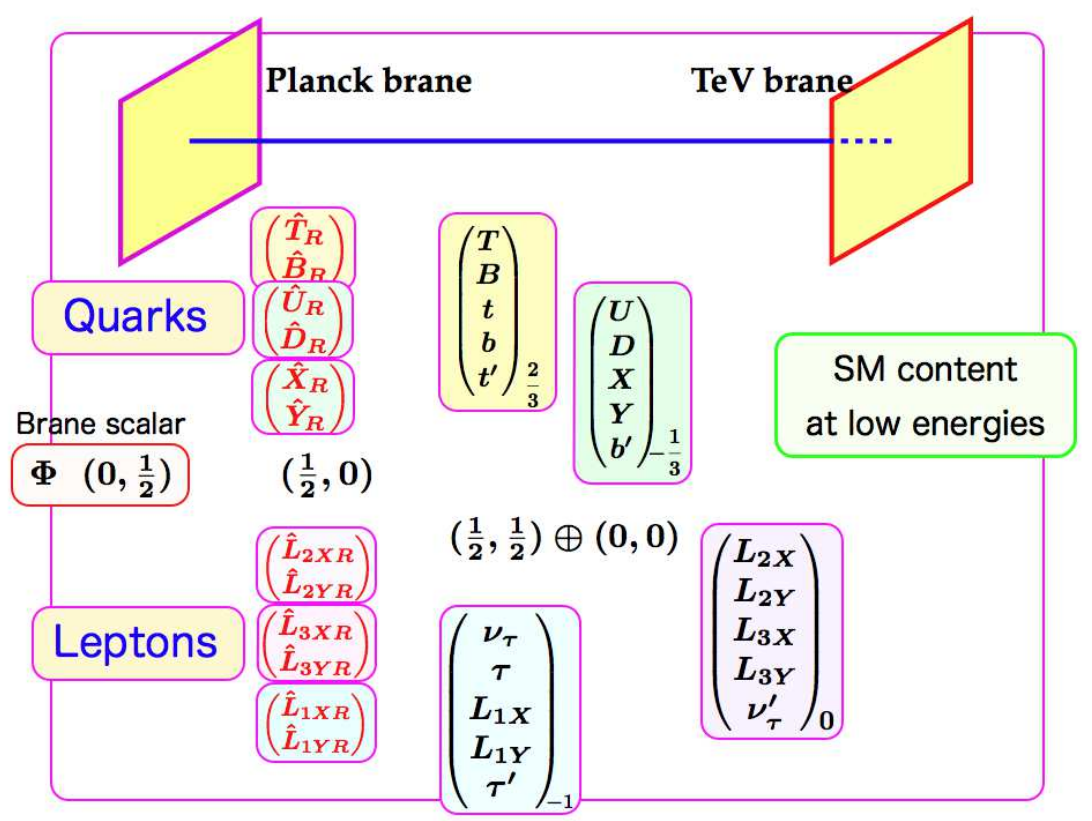

Figure 3: Matter content in the model.

where the zero-mode wave function is $h_{0}(y)=\left[2 k /\left(z_{L}^{2}-1\right)\right]^{1 / 2} e^{2 k y}(0 \leq y \leq L)$. The generator $T^{\hat{4}}$ is given by $\left(T_{\mathrm{vec}}^{\hat{4}}\right)_{a b}=(i / \sqrt{2})\left(\delta_{a 5} \delta_{b 4}-\delta_{a 4} \delta_{b 5}\right)$ in the vectorial representation and $T_{\mathrm{sp}}^{\hat{4}}=(1 / 2 \sqrt{2}) I_{2} \otimes \tau_{1}$ in the spinorial representation. $\hat{\theta}_{H}(x)$ is decomposed as

$$
\hat{\theta}_{H}(x)=\theta_{H}+\frac{H(x)}{f_{H}}, f_{H}=\frac{2}{g_{A}} \sqrt{\frac{k}{z_{L}^{2}-1}} \sim \frac{2}{\sqrt{k L}} \frac{m_{\mathrm{KK}}}{\pi g} .
$$

Here the Kaluza-Klein (KK) mass scale is $m_{\mathrm{KK}}=\pi k z_{L}^{-1}$, and the $S O(5)$ gauge coupling $g_{A}$ is related to the $4 \mathrm{D} S U(2)_{L}$ weak coupling $g$ by $g=g_{A} / \sqrt{L}$.

With $H(x)=0$, in the spinorial representation,

$$
P \exp \left\{i g_{A} \int_{0}^{L} d y A_{y}\right\}=\exp \left\{\frac{i}{2} \theta_{H} I_{2} \otimes \tau_{1}\right\}
$$

so that the constant part $\theta_{H}$ of $\hat{\theta}_{H}(x)$ represents an Aharonov-Bohm phase in the extra-dimension. $\hat{\theta}_{H}$ is a phase variable. There remains the residual gauge invariance $A_{M} \rightarrow A_{M}^{\prime}=\Omega A_{M} \Omega^{-1}-\left(i / g_{A}\right) \Omega \partial_{M} \Omega^{-1}$ which preserves the boundary conditions (2) and (3). (7, 15] In general, new gauge potentials satisfy new boundary conditions given by $P_{j}^{\prime}=\Omega\left(x, y_{j}-y\right) P_{j} \Omega\left(x, y_{j}+y\right)^{-1}$. The residual gauge invariance is defined with $\Omega(x, y)$ satisfying $P_{j}^{\prime}=P_{j}$. Consider a large gauge transformation

$$
\Omega^{\mathrm{large}}(y ; \alpha)=\exp \left\{-i \alpha \int_{0}^{y} d y \sqrt{4 k /\left(z_{L}^{2}-1\right)} h_{0}(y) \cdot T^{\hat{4}}\right\} .
$$


which shifts $\hat{\theta}_{H}(x)$ to $\hat{\theta}_{H}^{\prime}(x)=\hat{\theta}_{H}(x)+\alpha$. With $\alpha=2 \pi, P_{j}^{\prime}=P_{j}$ and $\hat{\theta}_{H}^{\prime}=\hat{\theta}_{H}+2 \pi$. It implies that physics is invariant under

$$
\theta_{H} \rightarrow \theta_{H}+2 \pi
$$

\section{Effective theory}

Four-dimensional fluctuations of the $\mathrm{AB}$ phase $\theta_{H}$ correspond to the $4 \mathrm{D}$ neutral Higgs field $H(x)$. In the effective theory of the low energy fields the Higgs field $H(x)$ enters always in the combination of $\hat{\theta}_{H}(x)$ in (5). The effective Lagrangian must be invariant under $\hat{\theta}_{H}(x) \rightarrow \hat{\theta}_{H}(x)+2 \pi$. The effective Higgs interactions with the $W, Z$ bosons, quarks and leptons at low energies are summarized as 4., 14, 16, 17.

$$
\mathcal{L}_{\text {eff }}=-V_{\text {eff }}\left(\hat{\theta}_{H}\right)-m_{W}^{2}\left(\hat{\theta}_{H}\right) W_{\mu}^{\dagger} W^{\mu}-\frac{1}{2} m_{Z}^{2}\left(\hat{\theta}_{H}\right) Z_{\mu} Z^{\mu}-\sum_{a, b} m_{a b}^{F}\left(\hat{\theta}_{H}\right) \bar{\psi}_{a} \psi_{b} .
$$

$V_{\text {eff }}\left(\hat{\theta}_{H}\right)$ is the effective potential which arises at the one loop level. It is finite and independent of the cutoff. [1] From the second derivative at the global minimum a finite Higgs mass $m_{H}$ is obtained. The finiteness of $m_{H}^{2}$ gives a solution to the gauge-hierarchy problem. [18]

The mass functions $m_{W}\left(\hat{\theta}_{H}\right), m_{Z}\left(\hat{\theta}_{H}\right)$ and $m_{a b}^{F}\left(\hat{\theta}_{H}\right)$ arise at the tree level. It is essential to include contributions coming from KK excited states in intermedium states. The effective Lagrangian (9) is obtained after integrating out all heavy KK modes.

In the $S O(5) \times U(1)$ model under consideration these mass functions are found to be, to good accuracy in the warped space, [10, 11, 14]

$$
\begin{aligned}
& \text { gauge-Higgs } \\
& \text { [SM ] } \\
& m_{W}\left(\hat{\theta}_{H}\right) \sim \frac{1}{2} g f_{H} \sin \hat{\theta}_{H}, \quad\left[\frac{1}{2} g(v+H)\right], \\
& m_{Z}\left(\hat{\theta}_{H}\right) \sim \frac{1}{2 \cos \theta_{W}} g f_{H} \sin \hat{\theta}_{H}, \quad\left[\frac{1}{2 \cos \theta_{W}} g(v+H)\right], \\
& m_{a b}^{F}\left(\hat{\theta}_{H}\right) \sim y_{a b}^{F} f_{H} \sin \hat{\theta}_{H}, \quad\left[y_{a b}^{F}(v+H)\right] .
\end{aligned}
$$

We have listed the formulas in the standard model in brackets on the right. It is seen that $v+H$ in the standard model is replaced approximately by $f_{H} \sin \hat{\theta}_{H}$ in the gauge-Higgs unification. In the standard model the mass functions are linear in the Higgs field $H$, whereas they become periodic, nonlinear functions of $H$ in the gauge-Higgs unification. It is a consequence of the phase nature of $\theta_{H}$. In other words the gauge invariance in the warped space naturally leads to the nonlinear behavior. 
The masses of the $W, Z$ bosons and fermions are given by $m_{W}=\frac{1}{2} g f_{H} \sin \theta_{H}$, $m_{Z}=m_{W} / \cos \theta_{W}$, and $m_{a b}^{F}=y_{a b}^{F} f_{H} \sin \theta_{H}$. An immediate consequence is that the Higgs couplings to $W, Z$, and fermions deviate from those in the standard model. In particular,

$$
\begin{aligned}
\left(\begin{array}{c}
W W H \\
Z Z H \\
\text { Yukawa }
\end{array}\right) & =\mathrm{SM} \times \cos \theta_{H}, \\
\left(\begin{array}{c}
W W H H \\
Z Z H H
\end{array}\right) & =\mathrm{SM} \times \cos 2 \theta_{H} .
\end{aligned}
$$

As we shall see below, the effective potential is minimized at $\theta_{H}=\frac{1}{2} \pi$ so that the $W W Z, Z Z H$ and Yukawa couplings vanish.

\section{Dynamical EW symmetry breaking}

The value of $\theta_{H}$ in the vacuum is determined by the location of the global minimum of the effective potential $V_{\text {eff }}\left(\theta_{H}\right)$, which is given at the one loop level by

$$
V_{\text {eff }}\left(\theta_{H}\right)=\sum_{\text {particles }} \pm \frac{1}{2} \int \frac{d^{4} p}{(2 \pi)^{4}} \sum_{n} \ln \left(p^{2}+m_{n}\left(\theta_{H}\right)^{2}\right)
$$

where $\left\{m_{n}\left(\theta_{H}\right)\right\}$ is a $4 \mathrm{D}$ mass spectrum in each $\mathrm{KK}$ tower with the $\mathrm{AB}$ phase $\theta_{H}$. As originally shown in ref. 1 the $\theta_{H}$-dependent part of $V_{\text {eff }}\left(\theta_{H}\right)$ is finite, independent of how the theory is regularized. Evaluation of $V_{\text {eff }}\left(\theta_{H}\right)$ in the RS warped space was initiated by Oda and Weiler. 19] Since then a powerful method for evaluation has been developed by Falkowski. 20.

In the model under consideration the electroweak symmetry $S U(2)_{L} \times U(1)_{Y}$ remains unbroken for $\theta_{H}=0, \pi$. Otherwise the symmetry is broken to $U(1)_{\mathrm{EM}}$. If there were no fermions, the symmetry is unbroken. Among the fermions, multiplets in the bulk containing the top quark gives a dominant contribution to $V_{\text {eff }}\left(\theta_{H}\right)$. The fact that the top quark mass $(172 \mathrm{GeV})$ is larger than $m_{W}$ is relevant. Contributions from other fermions, whose masses are much smaller than $m_{W}$, are negligible in the warped space. Contributions fom charm quarks, for instance, are suppressed by a factor $10^{-5}$. The existence of the top quark triggers the electroweak symmetry breaking. $U\left(\theta_{H}\right)=\left(16 \pi^{4} / m_{\mathrm{KK}}^{4}\right) V_{\text {eff }}\left(\theta_{H}\right)$ is plotted with the warp factor $z_{L}=10^{10}$ in fig. 4.

It is seen that the effective potential is minimized at $\theta_{H}= \pm \frac{1}{2} \pi$ so that the electroweak symmetry is dynamically broken. It follows from (10) that $m_{W}=$ $\frac{1}{2} g f_{H}$ and $f_{H} \sim 256 \mathrm{GeV}$.

As the warp factor $z_{L}=e^{k L}$ is decreased, there appear two kinds of phase transitions. For $z_{L}<z_{L 1} \sim 2200$ the top quark mass $m_{t} \sim 171 \mathrm{GeV}$ cannot be achieved. It necessarily becomes smaller than the observed value. One can set the bulk mass parameter to be zero, and further decrease the value of $z_{L}$. Then, for 


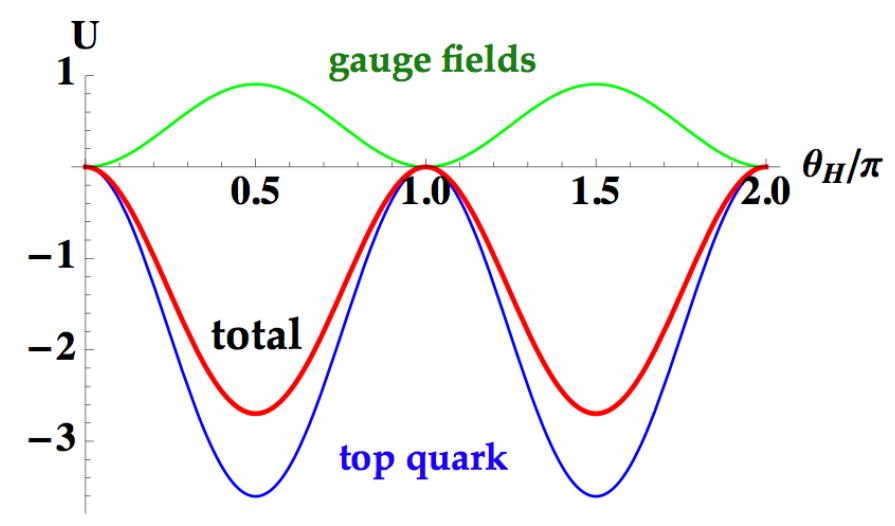

Figure 4: Effective potential $V_{\mathrm{eff}}\left(\theta_{H}\right)=m_{\mathrm{KK}}^{4} /\left(16 \pi^{4}\right) U\left(\theta_{H}\right)$ with $z_{L}=10^{10}$.

$z_{L} \sim z_{L 2} \sim 1.67$ there appears a weakly-first-order phase transition. For $z_{L}<z_{L 2}$ the global minima move to $\theta_{H}=0$ and $\pi$ so that the symmetry is unbroken.2

The curvature of the $V_{\text {eff }}$ at the minimum is related to the Higgs mass $m_{H}$ by

$$
m_{H}^{2}=\left.\frac{\pi^{2} g^{2} k L}{4 m_{\mathrm{KK}}^{2}} \frac{d^{2} V_{\mathrm{eff}}}{d \theta_{H}^{2}}\right|_{\min }, \quad m_{\mathrm{KK}}=\pi k z_{L}^{-1} .
$$

For $z_{L}=10^{10}, m_{\mathrm{KK}}=1.2 \mathrm{TeV}$ and $m_{H}=108 \mathrm{GeV}$. At first sight one might think that this value for $m_{H}$ contradicts with the LEP2 bound $m_{H}>114 \mathrm{GeV}$. However, the effective potential is minimized at $\theta_{H}= \pm \frac{1}{2} \pi$ where the $Z Z H$ coupling vanishes. See (111). The LEP2 bound is evaded as the process $e^{+} e^{-} \rightarrow Z^{*} \rightarrow Z H$ does not take place in the present model.

\section{The absolute stability of the Higgs bosons}

In the present gauge-Higgs unification model the $\mathrm{AB}$ phase is dynamically chosen to be $\theta_{H}=\frac{1}{2} \pi$ at the one loop level. It implies that all $Z Z H, W W H$ and Yukawa couplings of quarks and leptons vanish so that the Higgs boson cannot decay. Can the Higgs boson decay at higher orders? We show that the Higgs boson becomes absolutely stable in a class of the gauge-Higgs unification models including the present model. 4 .

\section{(i) Mirror reflection symmetry}

The theory is invariant under the mirror reflection in the fifth dimension;

$$
\begin{aligned}
\left(x^{\mu}, y\right) & \rightarrow\left(x^{\mu \prime}, y^{\prime}\right)=\left(x^{\mu},-y\right), \\
A_{M}(x, y) & \rightarrow A_{M}^{\prime}\left(x^{\prime}, y^{\prime}\right)=\left(A_{\mu},-A_{y}\right)(x, y), \\
\Psi_{a}(x, y) & \rightarrow \Psi_{a}^{\prime}\left(x^{\prime}, y^{\prime}\right)= \pm \gamma^{5} \Psi_{a}(x, y) .
\end{aligned}
$$

\footnotetext{
${ }^{2}$ There was a numerical error in the evaluation of $V_{\text {eff }}\left(\theta_{H}\right)$ in ref. 13 . .
} 
It implies that physics is invariant under

$$
\hat{\theta}_{H}(x)=\theta_{H}+\frac{H(x)}{f_{H}} \rightarrow \hat{\theta}_{H}^{\prime}\left(x^{\prime}\right)=-\hat{\theta}_{H}(x)
$$

while all other SM particles remain unchanged.

\section{(ii) Enhanced gauge invariance}

One may notice that the effective potential $V_{\text {eff }}\left(\theta_{H}\right)$ at the one loop level depicted in fig. (4 has periodicity $\pi$. Indeed, this remains true to all order. Gauge invariance is enhanced.

Consider a lare gauge transformation (7) with $\alpha=\pi$. With $\Omega^{\text {large }}(y ; \pi)$ the boundary conditions change to $\left(P_{0}^{\prime \text { vec }}, P_{1}^{\prime \text { vec }}\right)=\left(P_{0}{ }^{\text {vec }}, P_{1}{ }^{\text {vec }}\right)$ in the vectorial representation and $\left(P_{0}^{\prime \mathrm{sp}}, P_{1}^{\prime \mathrm{sp}}\right)=\left(P_{0}^{\mathrm{sp}},-P_{1}{ }^{\mathrm{sp}}\right)$ in the spinorial representation. The $\mathrm{AB}$ phase changes to $\theta_{H}^{\prime}=\theta_{H}+\pi$. In the present model all bulk fermions are in the vector representation. The brane fermions and scalar field on the Planck brane are not affected by this transformation as $\Omega^{\text {large }}(0 ; \pi)=1$. Hence the theory is invariant under $\theta_{H} \rightarrow \theta_{H}+\pi$. All physical quantities become periodic in $\theta_{H}$ with a reduced period $\pi$.

\section{(iii) H-parity}

In a class of the $S O(5) \times U(1)$ gauge-Higgs unification models in the warped space which contains bulk fermions only in tensorial representations of $S O(5)$ and brane fermions only on the Planck brane, the enhanced gauge symmetry with the mirror reflection symmetry leads to

$$
\begin{aligned}
& V_{\mathrm{eff}}\left(\hat{\theta}_{H}+\pi\right)=V_{\mathrm{eff}}\left(\hat{\theta}_{H}\right)=V_{\mathrm{eff}}\left(-\hat{\theta}_{H}\right), \\
& m_{W, Z}^{2}\left(\hat{\theta}_{H}+\pi\right)=m_{W, Z}^{2}\left(\hat{\theta}_{H}\right)=m_{W, Z}^{2}\left(-\hat{\theta}_{H}\right), \\
& m_{a b}^{F}\left(\hat{\theta}_{H}+\pi\right)=-m_{a b}^{F}\left(\hat{\theta}_{H}\right)=m_{a b}^{F}\left(-\hat{\theta}_{H}\right) .
\end{aligned}
$$

$m_{W, Z}(0)=m_{a b}^{F}(0)=0$ as the EW symmetry is recovered at $\theta_{H}=0$.

In the previous section we have seen that $V_{\text {eff }}\left(\theta_{H}\right)$ is minimized at $\theta_{H}=\frac{1}{2} \pi$. It follows then from (16) that all of $V_{\text {eff }}\left(\hat{\theta}_{H}\right), m_{W, Z}^{2}\left(\hat{\theta}_{H}\right)$ and $m_{a b}^{F}\left(\hat{\theta}_{H}\right)$ satisfy a relation $F\left(\frac{1}{2} \pi+f_{H}^{-1} H\right)=F\left(\frac{1}{2} \pi-f_{H}^{-1} H\right)$. They are even functions of $H$ when expanded around $\theta_{H}= \pm \frac{1}{2} \pi$. All odd-power Higgs couplings $H^{2 \ell+1}, H^{2 \ell+1} W_{\mu}^{\dagger} W^{\mu}$, $H^{2 \ell+1} Z_{\mu} Z^{\mu}$, and $H^{2 \ell+1} \bar{\psi}_{a} \psi_{b}$, vanish.

The effective interactions at low energies are invariant under $H(x) \rightarrow-H(x)$ with all other fields kept intact at $\theta_{H}= \pm \frac{1}{2} \pi$. There arises the $H$-parity invariance. Among low energy fields only the Higgs field is $H$-parity odd. The Higgs boson becomes absolutely stable, protected by the $H$-parity conservation.

\section{Stable Higgs bosons as cold dark matter}

Abosolutely stable Higgs bosons become cold dark matter (CDM) in the present universe. They are copiously produced in the very early universe. As the annihilation rate of Higgs bosons falls below the expansion rate of the universe, the 
annihilation processes get effectively frozen and the remnant Higgs bosons become dark matter. 4

The annihilation rates can be estimated from the effective Lagrangian (9) with (10). At $\theta_{H}=\frac{1}{2} \pi$ one has

$$
\mathcal{L}_{\text {eff }} \sim-\left\{m_{W}^{2} W_{\mu}^{\dagger} W^{\mu}+\frac{1}{2} m_{Z}^{2} Z_{\mu} Z^{\mu}\right\} \cos ^{2} \frac{H}{f_{H}}-\sum_{a} m_{a} \bar{\psi}_{a} \psi_{a} \cos \frac{H}{f_{H}} .
$$

As $m_{W} \sim \frac{1}{2} g f_{H}, f_{H}$ is determined to be $\sim 246 \mathrm{GeV}$. Although the Yukawa coupling $\bar{\psi}_{a} \psi_{a} H$ vanishes, the $\bar{\psi}_{a} \psi_{a} H^{2}$ coupling is nonvanishing, given by $\left(m_{a} / 2 f_{H}^{2}\right) \bar{\psi}_{a} \psi_{a} H^{2}$. It is generated by two vertices $\psi_{a} \psi_{a}^{(n)} H$ where $\psi_{a}^{(n)}$ is the $n$-th KK excited state of $\psi_{a}$.

The Higgs mass $m_{H}$ is predicted in the present model in the range of $50 \mathrm{GeV}$ to $130 \mathrm{GeV}$, depending on the value of the warp factor. If $m_{H}>m_{W}$, the dominant annihilation modes are $H H \rightarrow W W, Z Z$. The rate is large so that the resultant relic abundance becomes very small. For $m_{H}<m_{W}$ the relevant annihilation modes are $H H \rightarrow W^{*} W^{*}, Z^{*} Z^{*}, b \bar{b}, c \bar{c}, \tau \bar{\tau}$, and $g g$. Here $W^{*}\left(Z^{*}\right)$ indicates virtual $W(Z)$ which subsequently decays into a fermion pair. Annihilation into a gluon $(g)$ pair takes place through a top quark loop.

The relic abundance of Higgs bosons is evaluated as a function of $m_{H}$. It is depicted in fig. 5. It is seen that $\Omega_{H} h^{2}$ determined from the WMAP data is reproduced in the gauge-Higgs unification with $m_{H} \sim 70 \mathrm{GeV}$. It is remarkable that the gauge-Higgs unification scenario gives $\Omega_{H} h^{2}$ in the just right order of magnitude for $20 \mathrm{GeV}<m_{H}<75 \mathrm{GeV}$. With $m_{H}=70 \mathrm{GeV}$, the freeze-out temperature is $T_{f} \sim 3 \mathrm{GeV}$. The relative contributions of the $H H \rightarrow W^{*} W^{*}$ and $b \bar{b}$ modes are $61 \%$ and $34 \%$, respectively.

If Higgs bosons constitute the cold dark matter of the universe, they can be detected by observing Higgs-nucleon elastic scattering process, $H N \rightarrow H N$. The relevant part of the effective interaction (17) is $\mathcal{L}_{\text {eff }}=\left(H^{2} / 2 f_{H}^{2}\right) \sum_{f} m_{f} \bar{f} f$. With QCD corrections taken into account the effective interaction for the Higgs-nucleon coupling becomes

$$
\mathcal{L}_{H N} \simeq \frac{2+7 f_{N}}{9} \frac{m_{N}}{2 f_{H}^{2}} H^{2} \bar{N} N
$$

where $f_{N}=\sum_{q=u, d, s} f_{q}^{N}$ and $\left\langle N\left|m_{q} \bar{q} q\right| N\right\rangle=m_{N} f_{q}^{N}$. With this coupling (18) the spin-independent (SI) Higgs-nucleon scattering cross section is found to be

$$
\sigma_{\mathrm{SI}} \simeq \frac{1}{4 \pi}\left(\frac{2+7 f_{N}}{9}\right)^{2} \frac{m_{N}^{4}}{f_{H}^{4}\left(m_{H}+m_{N}\right)^{2}},
$$

in the non-relativistic limit. There is ambiguity in the value of $f_{N}$. Our prediction is $\sigma_{S I} \simeq(1.2-2.7) \times 10^{-43} \mathrm{~cm}^{2}$ for $f_{N}=(0.1-0.3)$. The present experimental upper bounds for the spin-independent WIMP-nucleon cross sections come from CDMS II 21] and XENON10 22. From the recent CDMS II data $\sigma_{\mathrm{SI}} \lesssim 7 \times$ $10^{-44} \mathrm{~cm}^{2}$ at $90 \% \mathrm{CL}$ with the WIMP mass $70 \mathrm{GeV}$. With many uncertainties and ambiguity in the analysis taken into account, this does not necessarily mean that the present gauge-Higgs unification model is excluded. The next generation 


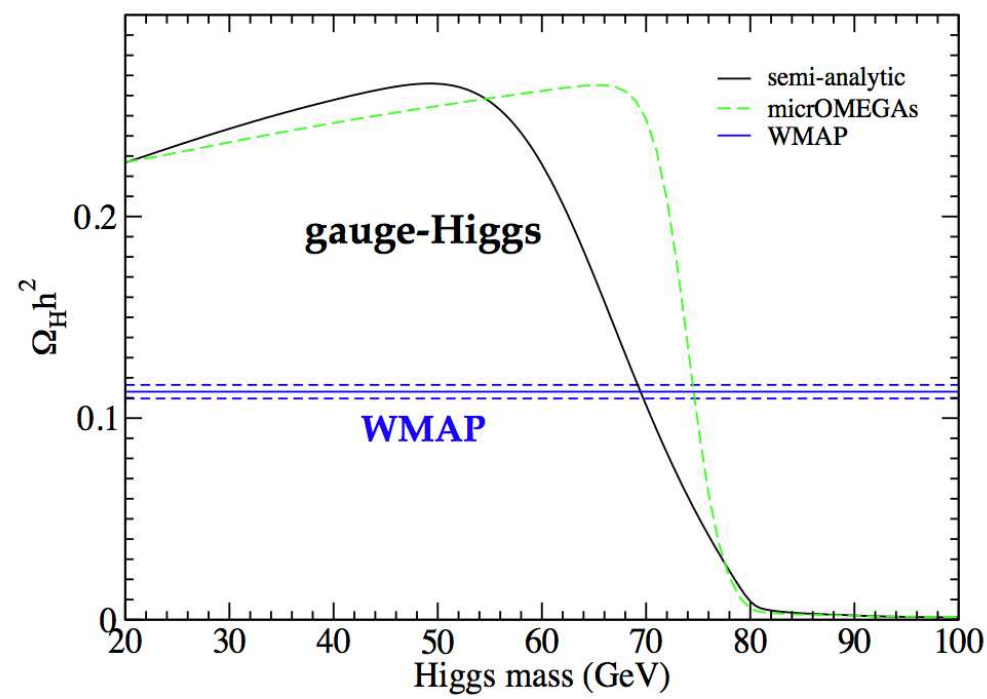

Figure 5: Thermal relic density of Higgs boson DM with $f_{H}=246 \mathrm{GeV}$. The solid curve is obtained by the semi-analytic formulae. The horizontal band is the WMAP data $\Omega_{\mathrm{CDM}} h^{2}=0.1131 \pm 0.0034$. The value in the WMAP data is obtained with $m_{H} \sim 70 \mathrm{GeV}$.

experiments for the direct detection of WIMP-nucleon scattering are awaited to pin down the rate.

\section{Stable Higgs bosons at Tevatron/LHC/ILC}

In all of the experiments performed so far, Higgs bosons are searched by trying to identify their decay products. If Higgs bosons are stable, however, this way of doing experiments becomes a vain effort.

Higgs bosons are produced in pairs. Typical processes are $Z^{*} \rightarrow Z H H$, $W^{*} \rightarrow W H H, W W \rightarrow H H, Z Z \rightarrow H H$, and $g g \rightarrow H H$. Higgs bosons are stable so that they appear as missing energies and momenta in collider experiments. The appearance of two particles of missing energies and momenta in the final state makes experiments hard, but not impossible. These events must be distinguished from those involving neutrinos. Since $m_{H} \sim 70 \mathrm{GeV}$, Higgs bosons can be copiously produced at LHC. It is a challenging task to identify stable Higgs bosons at colliders.

The effects of the vanishing $W W H$ and $Z Z H$ couplings can be seen in $W W$, $W Z$, and $Z Z$ elastic scattering, too. These scattering amplitudes become large as the energy is increased, much faster than in the standard model. 23. 


\section{Gauge couplings of quarks and leptons}

We have seen large deviations in the Higgs couplings from the standard model. There arise deviations in the gauge couplings of quarks and leptons as well, which have to be scrutinized with the electroweak precision measuremens. 5, 8, 9 ,

$5 \mathrm{D}$ gauge couplings of fermions are universal. However, 4D gauge couplings of quarks and leptons are obtained by inserting their wave functions and $W(Z)$ wave function into 5D Lagrangian and integrating over the fifth coordinate. Since the wave functions depend on quarks and leptons, slight variations appear in $4 \mathrm{D}$ gauge couplings, resulting in violation of the universality. The wave functions of $W$ and $Z$ bosons are determined in refs. [10] and [1], whereas those of quarks and leptons are determined in refs. [5] and [14].

There arise deviations in the $W$ boson couplings from the standard model. For the $t$ and $b$ quarks their interactions are given by

$$
\frac{1}{2} g_{t b, L}^{(W)}\left(W_{\mu} \bar{b}_{L} \gamma^{\mu} t_{L}+W_{\mu}^{\dagger} \bar{t}_{L} \gamma^{\mu} b_{L}\right)+\frac{1}{2} g_{t b, R}^{(W)}\left(W_{\mu} \bar{b}_{R} \gamma^{\mu} t_{R}+W_{\mu}^{\dagger} \bar{t}_{R} \gamma^{\mu} b_{R}\right) .
$$

Not only left-handed components but also right-handed components couple to $W$ in general. The $W$ coupling defined experimentally is the coupling between left-handed $e$ and $\nu_{e}, g_{e \nu, L}^{(W)}$. In the standard model the couplings are universal;

$g_{f, L}^{(W)}=g_{e \nu, L}^{(W)}$ and $g_{f, R}^{(W)}=0$. The violation of the universality for the left-handed quarks and leptons, $g_{f, L}^{(W)} / g_{e \nu, L}^{(W)}-1$, is summarized in Table 1 . The $W$ couplings to the right-handed quarks and leptons are summarized in Table 2. One can see that the deviation from the standard model is extremely tiny except for top-bottom, well below the current limits. For the left-handed top-bottom quarks the deviation is about $2 \%$.

Similarly the $Z$ boson couplings deviate from the standard model. The couplings of $t$ and $b$ quarks take the form

$$
\frac{1}{\cos \theta_{W}} Z_{\mu}\left\{g_{t L}^{(Z)} \bar{t}_{L} \gamma^{\mu} t_{L}+g_{t R}^{(Z)} \bar{t}_{R} \gamma^{\mu} t_{R}+g_{b L}^{(Z)} \bar{b}_{L} \gamma^{\mu} b_{L}+g_{b R}^{(Z)} \bar{b}_{R} \gamma^{\mu} b_{R}\right\} .
$$

The relevant couplings are $g_{f, L R}^{(Z)} / g_{e \nu, L}^{(W)}$. They are summarized in Table 3, in which the couplings in the standard model are also listed for comparison.

It is seen from the table, the deviation from the standard model is rather small ( $0.1 \%$ to $1 \%$ ) except for the top quark coupling for $z_{L}=10^{15}$. The $Z$ couplings of the left- and right-handed top quark deviate from those in the standard model

Table 1: The deviation of the $W$ couplings for left-handed leptons and quarks from the standard model for the warp factor $z_{L}=10^{15}$.

\begin{tabular}{|ccccc|}
\multicolumn{5}{c}{$g_{f L}^{(W)} / g_{e \nu, L}^{(W)}-1$} \\
\hline$\nu_{\mu}, \mu$ & $\nu_{\tau}, \tau$ & $u, d$ & $c, s$ & $t, b$ \\
\hline$-1.022 \times 10^{-8}$ & $-2.736 \times 10^{-6}$ & $-2.778 \times 10^{-11}$ & $-1.415 \times 10^{-6}$ & -0.02329 \\
\hline
\end{tabular}


Table 2: The $W$ couplings for right-handed leptons and quarks for the warp factor $z_{L}=10^{15}$. They are all tiny.

\begin{tabular}{|ccc|}
\multicolumn{3}{c}{$g_{f R}^{(W)} / g_{e \nu, L}^{(W)}$} \\
\hline$\nu_{e}, e$ & $\nu_{\mu}, \mu$ & $\nu_{\tau}, \tau$ \\
\hline$-4.761 \times 10^{-21}$ & $-4.141 \times 10^{-16}$ & $-1.133 \times 10^{-13}$ \\
\hline \hline$u, d$ & $c, s$ & $t, b$ \\
\hline$-1.392 \times 10^{-11}$ & $-1.896 \times 10^{-7}$ & -0.001264 \\
\hline
\end{tabular}

Table 3: The deviation of the $Z$ couplings for leptons and quarks from the standard model for the warp factor $z_{L}=10^{15}$. In the column SM the values in the standard model are quoted.

$$
\tilde{g}_{f, L R}^{(Z)}=g_{f, L R}^{(Z)} / g_{e \nu, L}^{(W)}
$$

\begin{tabular}{|c|ccc|c|}
\hline$f$ & $\nu_{e}$ & $\nu_{\mu}$ & $\nu_{\tau}$ & SM \\
\hline$\tilde{g}_{f, L}^{(Z)}$ & 0.500822 & 0.500822 & 0.500822 & 0.5 \\
\hline$\tilde{g}_{f R}^{(Z)}$ & $-5.754 \times 10^{-31}$ & $-5.392 \times 10^{-29}$ & $-1.840 \times 10^{-27}$ & 0 \\
\hline
\end{tabular}

\begin{tabular}{|c|ccc|c|}
\hline & $e$ & $\mu$ & $\tau$ & \\
\hline$\tilde{g}_{f L}^{(Z)}$ & -0.2692 & -0.2692 & -0.2692 & -0.2688 \\
\hline$\tilde{g}_{f R}^{(Z)}$ & 0.2334 & 0.2333 & 0.2333 & 0.2312 \\
\hline \hline & $u$ & $c$ & $t$ & \\
\hline$\tilde{g}_{f L}^{(Z)}$ & 0.3464 & 0.3464 & 0.3204 & 0.3459 \\
\hline$\tilde{g}_{f R}^{(Z)}$ & -0.1556 & -0.1556 & -0.1823 & -0.1541 \\
\hline
\end{tabular}

\begin{tabular}{|c|ccc|c|}
\hline & $d$ & $s$ & $b$ & \\
\hline$\tilde{g}_{f L}^{(Z)}$ & -0.4236 & -0.4236 & -0.4241 & -0.4229 \\
\hline$\tilde{g}_{f R}^{(Z)}$ & 0.07779 & 0.07777 & 0.07774 & 0.07707 \\
\hline
\end{tabular}

by $-7 \%$ and $18 \%$, respectively. The deviations in the $Z b_{L} \bar{b}_{L}$ and $Z b_{R} \bar{b}_{R}$ couplings are $0.3 \%$ and $0.9 \%$, respectively.

There results an important prediction for the forward-backward asymmetry in the $e^{+} e^{-}$collisions on the $Z$ pole as pointed out by Uekusa. 24. The asymmetry is given by

$$
A_{F B}^{f}=\frac{3}{4} A_{L R}^{e} A_{L R}^{f} \quad, \quad A_{L R}^{f}=\frac{\left(g_{f L}^{(Z)}\right)^{2}-\left(g_{f R}^{(Z)}\right)^{2}}{\left(g_{f L}^{(Z)}\right)^{2}+\left(g_{f R}^{(Z)}\right)^{2}} .
$$

The predictions are summarized in Table 4. It is exciting that the gauge-Higgs unification model gives better fits to the observed data for the asymmetry in $b \bar{b}$ and $c \bar{c}$ than the standard model. CP violation, anomalous magnetic moment, and electric dipole moment in gauge-Higgs unification have been also discussed. 25] 
Table 4: The forward-backward asymmetry on the $Z$ pole in $e^{+} e^{-}$collisions. The numbers in the gauge-Higgs unification scenario with the warp factor $z_{L}=10^{15}$ are quoted from Uekusa, ref. 24.

$$
A_{F B}^{f}
$$

\begin{tabular}{|c|c|c|c|}
\hline$f$ & Experiment & Gauge-Higgs & Standard Model \\
\hline$c$ & $0.0707 \pm 0.0035$ & 0.07073 & $0.0738 \pm 0.0006$ \\
\hline$s$ & $0.0976 \pm 0.0114$ & 0.09950 & $0.1034 \pm 0.0007$ \\
\hline$b$ & $0.0992 \pm 0.0016$ & 0.09952 & $0.1033 \pm 0.0007$ \\
\hline$e$ & $0.0145 \pm 0.0025$ & 0.01511 & $0.01627 \pm 0.00023$ \\
\hline$\mu$ & $0.0169 \pm 0.0013$ & 0.01513 & \\
\hline$\tau$ & $0.0188 \pm 0.0017$ & 0.01515 & \\
\hline
\end{tabular}

\section{Summary}

The $S O(5) \times U(1)$ gauge-Higgs unification in the Randall-Sundrum warped spacetime is promising. The Higgs boson becomes a part of the gauge fields in higher dimensions, and is unified with gauge bosons. The resultant 4D gauge couplings of quarks and leptons are close to those in the standard model. The Higgs couplings, on the other hand, deviate significantly from those in the standard model.

In a large class of the $S O(5) \times U(1)$ gauge-Higgs unification models the Higgs boson becomes absolutely stable to all order in perturbation theory. In the evolution of the universe Higgs bosons become cold dark matter. From the WMAP data the Higgs boson mass is determined to be around $70 \mathrm{GeV}$.

In collider experiments Higgs bosons are produced in pairs. They appear as missing energies and momenta. The way of searching for Higgs bosons must be altered.

\section{Acknowledgment}

This work was supported in part by Scientific Grants from the Ministry of Education and Science, Grant No. 20244028, Grant No. 20025004, and Grant No. 50324744 .

\section{References}

[1] Y. Hosotani, Phys. Lett. B 126, 309 (1983).

[2] Y. Hosotani, Annals Phys. 190, 233 (1989).

[3] A. T. Davies and A. McLachlan, Phys. Lett. B 200, 305 (1988); Nucl. Phys. B 317, 237 (1989).

[4] Y. Hosotani, P. Ko and M. Tanaka, Phys. Lett. B 680, 179 (2009) arXiv:0908.0212 [hep-ph]].

[5] Y. Hosotani, S. Noda, and N. Uekusa, arXiv:0912.1173 [hep-ph] (to appear in Prog. Theoret. Phys.). 
[6] R. Contino, Y. Nomura and A. Pomarol, Nucl. Phys. B 671, 148 (2003) arXiv:hep-ph/0306259.

[7] Y. Hosotani and M. Mabe, Phys. Lett. B 615, 257 (2005) arXiv:hep-ph/0503020.

[8] K. Agashe, R. Contino and A. Pomarol, Nucl. Phys. B 719, 165 (2005) arXiv:hep-ph/0412089.

[9] Y. Hosotani, S. Noda, Y. Sakamura and S. Shimasaki, Phys. Rev. D 73, 096006 (2006) arXiv:hep-ph/0601241.

[10] Y. Sakamura and Y. Hosotani, Phys. Lett. B 645, 442 (2007) arXiv:hep-ph/0607236.

[11] Y. Hosotani and Y. Sakamura, Prog. Theor. Phys. 118, 935 (2007) arXiv:hep-ph/0703212.

[12] A. D. Medina, N. R. Shah and C. E. M. Wagner, Phys. Rev. D 76, 095010 (2007) arXiv:0706.1281 [hep-ph]].

[13] Y. Hosotani, K. Oda, T. Ohnuma and Y. Sakamura, Phys. Rev. D 78, 096002 (2008) [Erratum-ibid. D 79, 079902 (2009)] arXiv:0806.0480 [hep-ph]].

[14] Y. Hosotani and Y. Kobayashi, Phys. Lett. B 674, 192 (2009) arXiv:0812.4782 [hep-ph]].

[15] N. Haba, M. Harada, Y. Hosotani and Y. Kawamura, Nucl. Phys. B 657, 169 (2003) [Erratum-ibid. B 669, 381 (2003)] arXiv:hep-ph/0212035.

[16] Y. Sakamura, Phys. Rev. D 76, 065002 (2007) arXiv:0705.1334 [hep-ph]].

[17] G. F. Giudice, C. Grojean, A. Pomarol and R. Rattazzi, JHEP 0706, 045 (2007) arXiv:hep-ph/0703164.

[18] H. Hatanaka, T. Inami and C. S. Lim, Mod. Phys. Lett. A 13, 2601 (1998) arXiv:hep-th/9805067.

[19] K. Oda and A. Weiler, Phys. Lett. B 606, 408 (2005).

[20] A. Falkowski, Phys. Rev. D 75, 025017(2007).

[21] Z. Ahmed et al. [CDMS Collaboration], Phys. Rev. Lett. 102, 011301(2009) arXiv:0802.3530 [astro-ph]]; arXiv:0912.3592 [astro-ph.CO].

[22] J. Angle et al. [XENON Collaboration], Phys. Rev. Lett. 100, 021303(2008) arXiv:0706.0039 [astro-ph]].

[23] N. Haba, Y. Sakamura and T. Yamashita, arXiv:0908.1042 [hep-ph].

[24] N. Uekusa, arXiv:0912.1218 [hep-ph].

[25] Y. Adachi, C. S. Lim and N. Maru, Phys. Rev. D 76, 075009 (2007) arXiv:0707.1735 [hep-ph]]; Phys. Rev. D 80, 055025 (2009) arXiv:0905.1022 [hep-ph]]. 